\title{
The Future of Space Astrometry
}

\author{
Kenneth J. Johnston \\ United States Naval Observatory, Washington, D. C. 20392
}

\begin{abstract}
The future of space-based astrometric missions appears to be very promising. Three missions were described at IAU Colloquium 180. These are the Full-sky Astrometric Mapping Explorer (FAME), the Space Interferometry Mission (SIM) and the Global Astrometric Interferometer for Astrophysics (GAIA) missions. These missions will substantially improve the accuracy of global astrometric measurements made by the Hipparcos space mission by factors of 20 to 250 . A brief summary of these projects is given.
\end{abstract}

\section{Introduction}

This paper summarizes the presentations made at IAU Colloquium 180 on future space astrometry missions. These papers were presented by K. J. Johnston, F. Mignard and R. Danner for the FAME, GAIA and SIM missions respectively. Since these missions are at present only in the planning stages, changes will be made in the parameters that describe the missions in detail. This presentation is meant to give a very broad overview of these projects. The success of ESA's Hipparcos mission has shown that the accuracy of astrometric measurements can be greatly improved with space based instruments. Hipparcos improved the accuracy of global astrometric measurements from 50 to 1 milliarcseconds (mas). The Hipparcos mission used technologies available in the 1970s including a scanning image dissector tube to make the measurements. With advances in technology, namely CCDs, computers and interferometry, further improvements in accuracy can be made. Global astrometric accuracy of 50 to 4 microarcseconds ( $\mu$ as) is the aim of future missions. Figure 1 displays the present and expected accuracy that astrometric measurements have made and are expected to achieve in the next ten years.

\section{The missions}

There are two methods of measuring star positions. One can survey a large number of stars by continuously scanning a wide field of view or one can point at individual stars or a star field. In the first case, a large number of stars can be measured. The second case where a telescope stares at individual star fields, allows a longer integration time with the promise of achieving higher accuracy for fainter objects. The angular accuracy of the measurements is simply proportional to the inverse of the square root of the number of photons detected 


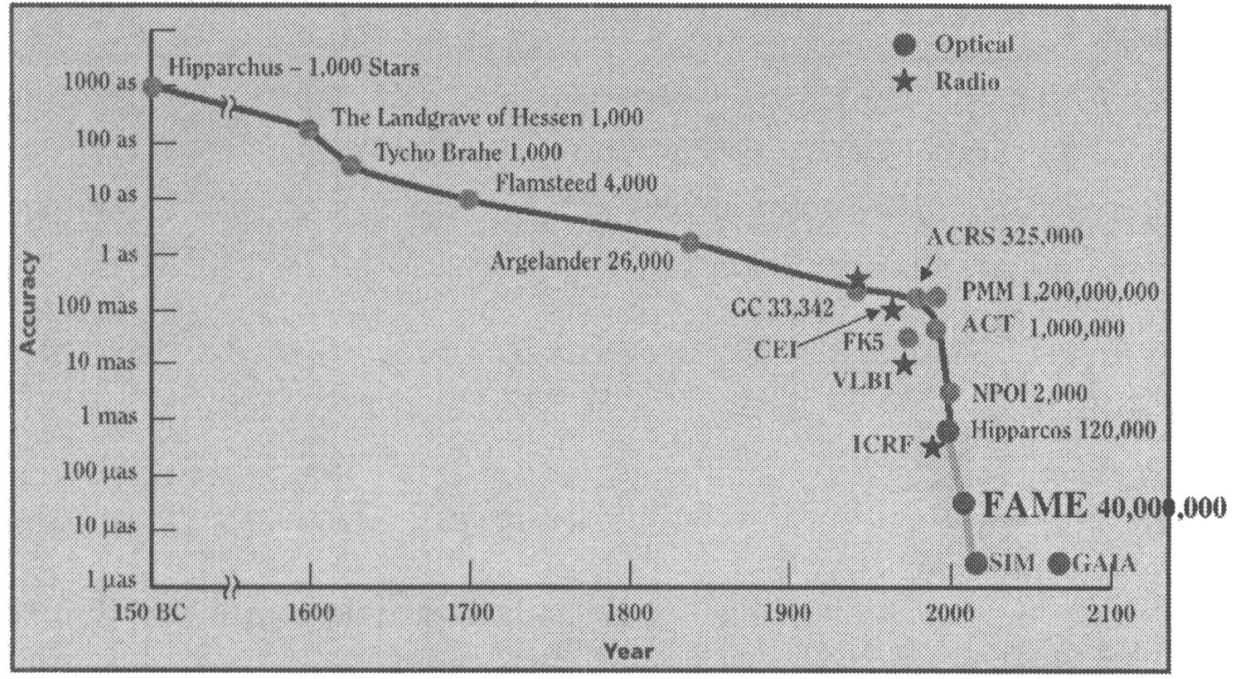

Figure 1. The increase in astrometric accuracy over large angle. Please note the great advance in accuracy achieved since the $1980 \mathrm{~s}$. 


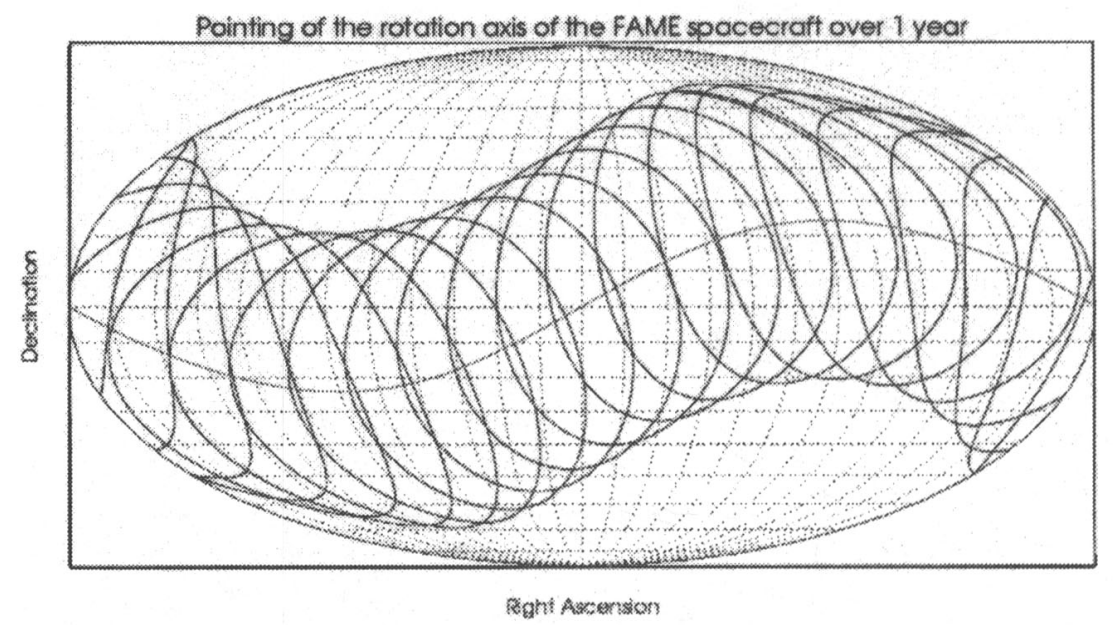

Pointing of FAME rotation axis - The spacecraft rotation axis precesses about the Sun with a 20 day period and a nominal Sun angle of $45^{\circ}$. The red line indicates the ecliptic.

Figure 2.

from the star times the point spread function of the telescope or synthesized beam of the interferometer.

Two of the proposed future missions trace their architecture directly to the Hipparcos mission. Instead of using an image dissector tube, a wide field of view of order of a degree or greater, with a focal plane filled with CCD arrays, is employed. The telescope scans the sky in a way similar to the Hipparcos mission using large $(2048 \times 1024$ pixels $)$ CCD arrays in a wide field of view focal plane, millions to a billion stars can be observed.

The FAME and GAIA missions are based on continuously scanning telescopes that look at two fields of view separated by fixed angles of 81.5 (FAME) and 106 (GAIA) degrees. The spacecraft rotates slowly with a rate of once every 40 minutes (FAME) and 180 minutes (GAIA). As it rotates, the spacecraft precesses around the Sun direction at a rate of 0.75 (FAME) and 0.17 (GAIA) arcseconds/second. In this way the spacecraft maps out the sky over a period of a year in a spiral pattern shown in Figure 2 for the FAME mission. The spacecraft will always look away from the Sun with the Sun-spacecraft rotation angle being 45 (FAME) and 55 (GAIA) degrees. The major difference in these two missions is the size of the telescope. FAME's proposed telescope aperture is 0.6 by $0.25 \mathrm{~m}$. GAIA's proposed telescope is $2 \mathrm{~m}$ in aperture. With the larger telescope aperture, GAIA's accuracy surpasses that of FAME.

FAME is an approved NASA MIDEX mission, selected in October, 1999. This five-year mission is scheduled for launch in June, 2004. A catalog will be 
available three and one half years after launch with accuracies listed in Table 1. The mission will measure the positions, parallax and proper motions of 40 million stars reaching stars as faint as to 15 th visual magnitude.

GAIA is a candidate for the ESA Cornerstone 5 mission, which will be selected in September, 2000. The projected accuracy is listed in Table 1. This mission has the capability to measure the positions of about a billion stars and will reach 20th visual magnitude. It is also a five-year mission.

Table 1.

$\begin{array}{lccc}\text { Mission } & \begin{array}{c}\text { Proposed } \\ \text { Launch Date }\end{array} & \text { No. of Stars } & \text { Global Accuracy } \\ \text { FAME } & \text { June 2004 } & 40 \times 10^{6} & 50 \mu \text { as @ } \mathrm{m}_{v}=9^{\text {th }} \\ \text { SIM } & 2007 & 10^{4} & 4 \mu \text { as @ } \mathrm{m}_{v}=12 \\ \text { GAIA } & 2009 & 10^{9} & 10 \mu \text { as @ } \mathrm{m}_{v}=15\end{array}$

The third mission is SIM. This mission points at individual stars and uses an interferometer to measure their positions. The interferometer consists of seven $35 \mathrm{~cm}$ siderostats distributed along a $10 \mathrm{~m}$ baseline. The interferometer will use two guide interferometers to determine the aspect of the instrument's baseline and a third interferometer to obtain the science data for the star observed. Since this is a pointed mission, long integration allows for improved accuracy over a scanning mission. This instrument will be capable of $4 \mu$ as accuracy for a 20th visual magnitude star. It is estimated that this mission will study about 10,000 objects. Another advantage of a pointed mission is that it can observe targets of opportunity in detail. Further, the interferometer will have a resolution of 10 mas while the FAME and GAIA missions will at best have a point spread function that is ten to five times larger than the resolution offered by SIM. This will be important in studying close binary stars. The mission accuracy reported in Table 1 is for global astrometry. SIM will have a narrow angle capability astrometric accuracy of 1 mas. The major impetus for this accuracy is the discovery of near terrestrial size extra-solar planets. The SIM mission is an integral part of NASA's Origins program. It is scheduled for a five-year mission with launch in Oct. 2008 timeframe, at this time. The Origins program also contains the Next Generation Space Telescope (NGST) and the Terrestrial Planet Finder (TPF) missions. The TPF mission will build on the results of SIM by studying in detail the planets discovered by SIM.

\section{Science}

All three missions have as their major objectives important science that will result from the improved accuracy in position, parallax and proper motions. Without this strong scientific justification, these missions could not go forward. The improvement in accuracy by a factor of 20 to 250 over the Hipparcos mission allows the volume of space over which stellar parameters can be precisely determined to increase dramatically. This goes as the cube of the improved accuracy. 
For example, the Hipparcos mission determined the distances to $10 \%$ accuracy for stars within 100 parsecs. The FAME mission will improve this by a factor of 20 resulting in fundamental knowledge of stellar masses, absolute luminosities, distances, and their motions in three-dimensional space for stars to distances of $2 \mathrm{kpc}$. This volume contains almost every type of star. The SIM and GAIA missions will be able to measure the distance to any object visible in the Milky Way Galaxy to $10 \%$ accuracy. The improved accuracy of these missions affects almost all areas of galactic astronomy. This will result in improved determination of the distance scale of the universe, detection of many extra-solar planetary systems, mapping the dark matter in the galactic disk, and improved knowledge of the evolution of stars.

It will also result in an improved celestial reference frame. The present International Celestial Reference Frame (ICRF) is based on the radio positions of extragalactic sources. The present accuracy of the positions of these sources is $250 \mu$ as. The optical positions of extragalactic sources can also be used to determine a reference frame from observations with the three space missions. The FAME mission will only reach a visual magnitude of 15 . This will limit the number of extragalactic reference sources to about fifty. The frame will be accurate at epoch to sub-microarcsecond accuracy based on star positions. The rotation of the frame will be uncertain to about $1 \mu \mathrm{as} / \mathrm{yr}$. The SIM and GAIA missions will be capable of observing quasars at visual magnitudes as faint as 20. All three missions will be capable of improving the value of the relativistic parameter of the Parameterized Post Newtonian (PPN) formulation of gravitational theory.

\section{Conclusion}

Space offers further improved accuracy in the determination of the positions, parallax and proper motion of stars. The science resulting from this improvement is driving several new missions. The three mission discussed are very complementary. The FAME mission will lead the way in the scientific endeavors discussed above. Its preliminary results will be available by 2007 . This will coincide with the launch of the SIM mission. Close coordination between these two missions will be necessary to maximize the scientific return. Interesting targets along with data on the stars making up the SIM grid will be available from the FAME mission. The improved accuracy of the SIM mission will allow for improved calibration of the FAME data. The GAIA mission will perform a survey with much improved accuracy that will extend the FAME survey to 20th visual magnitude. Since both of these missions are scanning missions, GAIA and FAME can benefit from sharing their technology development.

The capabilities of space astrometry are very promising. Extending the accuracy of astrometric measurements is critical to our future understanding of astrophysics. The missions described here will greatly extend our knowledge of galactic and stellar astrophysics. It is paramount that these missions take place. 


\section{References}

More up-to-date information on these missions may be obtained on their individual websites. These are:

FAME: http://aa.usno.navy.mil/FAME/

SIM: http://sim.jpl.nasa.gov

GAIA: http://astro.estec.esa.nl/GAIA/ 